Journal Club

Editor's Note: These short, critical reviews of recent papers in the Journal, written exclusively by graduate students or postdoctoral fellows, are intended to summarize the important findings of the paper and provide additional insight and commentary. For more information on the format and purpose of the Journal Club, please see http://www.jneurosci.org/misc/ifa_features.shtml.

\title{
From Dendritic Compartments to Neuronal Networks: A Multilevel Analysis of Motion Vision
}

\author{
Peter T. Weir ${ }^{1}$ and Marie P. Suver ${ }^{2}$ \\ ${ }^{1}$ Department of Biology, University of Washington, Seattle, Washington 98195, and ${ }^{2}$ Computation and Neural Systems, California Institute of Technology, \\ Pasadena, California 91125 \\ Review of Elyada et al.
}

Animals typically rely on vision to direct their locomotion through the environment. Flies, who move in three dimensions while in flight, have evolved the fastest visual system in the animal kingdom to help them stabilize their flight posture and trajectories (Autrum, 1958). Partly for this reason, they have been the subject of extensive research on the neuronal basis of motion vision, the component of visual function involved in detecting movement within a scene.

Using a variety of techniques, including electrophysiology, genetic manipulation, and behavioral analysis, researchers have started to unravel the earliest stages of motion processing (Clark et al., 2011; Eichner et al., 2011). Visual motion processing in the fly begins with the elementary motion detectors (EMDs), which are units sensitive to one direction of motion over a small receptive field. The identities of the cells involved in this computation are under active research, and a complete picture has yet to emerge. For over four decades, however, the identity of one set of downstream cells that receive input

Received April 17, 2013; revised May 10, 2013; accepted May 13, 2013.

This work was supported by grants from the Air Force Office of Scientific Research (FA9550-10-1-0368) and the Paul G. Allen Family Foundation (both to Michael H. Dickinson). We thank Bettina Schnell for useful conversations and Michael H. Dickinson for comments on the manuscript.

The authors declare no competing financial interests.

Correspondence should be addressed to Marie P. Suver, UW Biology, Box351800, Seattle,WA 98195-1800. E-mail:msuver@caltech.edu.

DOI:10.1523/JNEUROSCI.1634-13.2013

Copyright $\odot 2013$ the authors $\quad 0270-6474 / 13 / 339876-03 \$ 15.00 / 0$ from the EMDs has been known (Braitenberg, 1972). These cells, located in the lobula plate of the optic lobe of the fly, are called the horizontal system (HS) and vertical system (VS) cells.

First named for their conspicuous vertical and horizontal dendritic arbors, the VS and HS cells were later found to respond to visual motion in the vertical and horizontal directions, respectively. Their axonal responses are graded changes in membrane potential typically devoid of action potentials. The motion sensitivity of these cells arises from the integration of direct input from a retinotopic array of excitatory and inhibitory EMDs (Borst et al., 1995). These cells were initially thought to be most sensitive to movement in restricted fields of view. However, later studies showed that VS cells are responsive to motion across large regions in both the ipsilateral and contralateral visual fields, and respond best to motion that corresponds to the optic flow generated by the fly rotating about different body axes (Krapp and Hengstenberg, 1996).

For some time it was unclear how the receptive fields of VS and HS cells arise, given the anatomically limited range of their dendritic arbors. How does information from outside the receptive field of areas covered by the dendrites of a VS cell affect its axonal membrane potential? By using paired recordings, Haag and Borst (2004) demonstrated that the VS cells are connected via gap junctions into a network in which neighboring cells excite each other, broadening and tuning the receptive field of each. The rotational sensitivity of these cells is now attributed in part to the tuning properties of their dendritic inputs and in part to the connectivity pattern within the network. This finding complicated the former model of independently operating cells, and serves as a reminder that the function of nervous systems is inherently a network function. It cannot necessarily be divided neatly into upstream and downstream cellular components, but almost always includes lateral and recurrent connections as well.

This body of work detailing the construction of the VS and HS system's axonal output, combined with the ongoing research on the upstream input components, gives a reasonably complete picture of the input-output transformation of the system. An important aspect remains to be determined, however: how the transformation is implemented by processing in the dendritic compartments. For this, data on responses in different parts of the cell must be combined with accurate models to test the sufficiency of hypothesized mechanisms. Researchers have constructed multicompartment models of the VS-HS cell network that do reasonably well in recapitulating the output of the system, but are not fully compatible with all experimental observations of activity in the dendrites.

Elyada and colleagues (2013) recently monitored activity in the dendrites of VS and HS cells with a calcium-sensitive dye. 


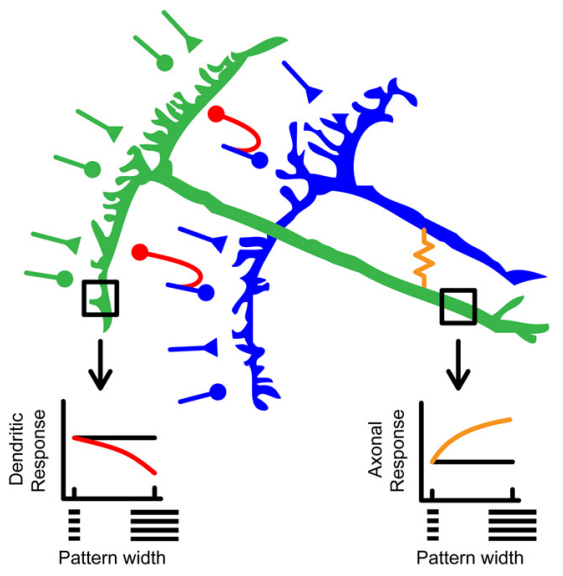

Figure 1. Schematic of end inhibition in a VS cell network. Two VS cells are shown in green (VS1) and blue (VS2). Direct excitatory (triangles) and inhibitory (circles) EMD inputs are depicted in the same colors. In the absence of end inhibition, the expected response in the dendrites is independent of the width of moving patterns (left inset, black line). End inhibitory input to VS1 is shown as red collaterals, originating from direct inhibitory EMD input in the receptive field of VS2, as supported by the current injection experiments. This input decreases the response to wider patterns (left inset, red line). In the axon, the expected response is independent of pattern width (right inset, black line). The inclusion of a gap junction between the axons of VS1 and VS2 (orange connection) results in increased responses to increasing pattern width (right inset, orange line). For simplicity, end inhibition in VS2 is not shown.

They reported a previously unknown phenomenon at work, termed "end inhibition," which consists of a decrease in dendritic responses when patterns are extended in the direction perpendicular to a cell's preferred direction of motion. Thus, the responses in the dendrites of a VS cell, sensitive to vertical motion, decrease when the same vertically moving pattern is made wider. This inhibition in the dendrites contrasts with the behavior at the axon of the same cell: because of gap junctions with neighboring VS cells, axonal responses increase when stimulus patterns extend over a wider horizontal area. These effects are illustrated in Figure 1.

Two questions immediately arise regarding end inhibition: what mechanism accounts for it, and what effect does it have on the behavior of the VS-HS cell network? In answering the former, Elyada et al. (2013) reasoned that end inhibition could act directly on the VS cells themselves by opening inhibitory channels, or alternatively, act upstream in the circuit by inhibiting the input EMDs and decreasing their synaptic release. If VS cells were directly inhibited, the opening of inhibitory channels would result in a decrease in cellular impedance, whereas an indirect decrease in synaptic drive would result in fewer open channels and an increase in cellular impedance during end inhibition. To distinguish the two possibilities, the researchers injected current into the VS cells while stimulating them with visual patterns of different widths. Unfortunately, the only example of raw data shows responses to a pattern of only one width, whereas end inhibition can only be observed by comparing responses to patterns of different widths. The authors report that increasing the stimulus width decreases cellular impedance, indicating that end inhibition is a result of inhibition by upstream cells acting directly on the VS cells. They suggest that the inhibitory input from upstream EMDs outside the VS cell's canonical receptive field are likely candidates for the source of this inhibition (Fig. 1, red curved inhibitory elements).

With evidence about where the end inhibition originates, Elyada et al. (2013) turned to the question of what impact it has on the function of the VS-HS cell network. A direct intervention to test this is technically infeasible: presumably, blocking end inhibitory elements would also disrupt the function of the EMDs that are responsible for the canonical operation of the VS and HS cells. Here, a multicompartmental model (Elyada et al., 2009) proved to be useful, allowing examination of the impact of end inhibition on responses to patterns of different widths. In the authors' model, each cell was represented by a dendritic and an axonal compartment, and a simple conductance represented the electrical connection between each pair of neighboring cells (Fig. 1, orange connection). During simulations, the dendritic compartments of the model received inputs from an array of inhibitory and excitatory EMD-like units acting on stimulus images. End inhibition was incorporated by replacing a fraction of the direct inhibitory inputs (inside the canonical receptive field of the cell) with inhibitory inputs that originally fed only into neighboring cells.

The authors make two principal points with their model. First, they tested the model's ability to recapitulate the experimental observations. Indeed, the model's dendritic responses decreased as pattern width increased, but its axonal responses increased as a function of width. Second, the authors found that end inhibition suppressed the responses of VS cells to both rotational and translational motion. Importantly, however, end inhibition increased the ratio of responses during rotation to responses during translation, thereby enhancing the relative sensitivity of the model to rotational flow fields. Hence, end inhibition appears to add to the rotational tuning of the VS-HS cell network.

This study raises challenges for neuroscientists accustomed to viewing neuronal function as monolithically represented by membrane potential, at least in these nonspiking cells. The work of Elyada and colleagues (2013) demonstrates that to understand the receptive fields of VS and HS cells, one must go beyond observations of their axonal output. Indeed, although the influence of end inhibition is unobservable at the axon terminals of these cells, the modeling work suggests that the receptive fields of those axons are fundamentally altered by its existence. Thus, to understand not only the input-output transformation of these cells, but also how that transformation is implemented, electrophysiology had to be combined with imaging and modeling.

The idea that single neurons contain different computational subunits is not a new one. Drawing from work across many organisms and cell types, it is now understood that computations in the dendrites of a neuron play a fundamental role in its overall function (London and Häusser, 2005). In locusts, for example, the output firing rate of the lobula giant movement detector neuron corresponds to an object moving on a collision course with the animal. This output is believed to be computed as a multiplication of inputs from distinct dendritic branches representing the speed of motion and the size of the moving object (Gabbiani et al., 2002). The accessibility of these dendrites for electrophysiological recordings made this characterization of dendritic computations possible. In preparations in which dendrites are not as easily accessible, calcium imaging is a powerful tool, as Elyada et al. (2013) show. In the future, technical advances may increase our ability to directly manipulate and elucidate computation in individual dendritic compartments. Furthermore, performing these studies in behaving animals will produce a more complete understanding of how single-cell computations ultimately affect an organism's behavior.

\section{References}

Autrum H (1958) Electrophysiological analysis of the visual systems in insects. Exp Cell Res 14:426-439. CrossRef Medline

Borst A, Egelhaaf M, Haag J (1995) Mechanisms of dendritic integration underlying gain control in fly motion-sensitive interneurons. J Comput Neurosci 2:5-18. CrossRef Medline Braitenberg V (1972) Periodic structures in the visual system of the fly. In: Information pro- 
cessing in the visual systems of arthropods (Wehner R, ed), pp 3-15. Berlin: Springer.

Clark DA, Bursztyn L, Horowitz MA, Schnitzer MJ, Clandinin TR (2011) Defining the computational structure of the motion detector in Drosophila. Neuron 70:1165-1177. CrossRef Medline

Eichner H, Joesch M, Schnell B, Reiff DF, Borst A (2011) Internal structure of the fly elementary motion detector. Neuron 70:11551164. CrossRef Medline

Elyada YM, Haag J, Borst A (2009) Different re- ceptive fields in axons and dendrites underlie robust coding in motion-sensitive neurons. Nat Neurosci 12:327-332. CrossRef Medline

Elyada YM, Haag J, Borst A (2013) Dendritic end inhibition in large-field visual neurons of the fly. J Neurosci 33:3659-3667. CrossRef Medline

Gabbiani F, Krapp HG, Koch C, Laurent G (2002) Multiplicative computation in a visual neuron sensitive to looming. Nature 420:320-324. CrossRef Medline

Haag J, Borst A (2004) Neural mechanism un- derlying complex receptive field properties of motion-sensitive interneurons. Nat Neurosci 7:628-634. CrossRef Medline

Krapp HG, Hengstenberg R (1996) Estimation of self-motion by optic flow processing in single visual interneurons. Nature 384: 463-466. CrossRef Medline

London M, Häusser M (2005) Dendritic computation. Annu Rev Neurosci 28:503-532. CrossRef Medline 